\title{
Effect of particle size, blend ratio and some selective bio-additives on rheological behaviour of high-concentration iron ore slurry
}

\author{
PK Senapati CSIR-Institute of Minerals and Materials Technology, India \\ JK Pothal CSIR-Institute of Minerals and Materials Technology, India \\ R Barik CSIR-Institute of Minerals and Materials Technology, India \\ R Kumar National Mineral Development Corporation, India
}

SK Bhatnagar Ministry of Steel, India

\begin{abstract}
The influence of particle size, blend ratio and some selective bio-additives on the rheological behaviour of an Indian iron ore sample in a slurry concentration range of $60-75 \%$ by mass was evaluated. The rheological parameters were measured using a high-precision ThermoFisher Scientific HAAKE ${ }^{T M}$ RheoStress ${ }^{T M} 1$ Rheometer. The iron ore slurry samples indicated non-Newtonian flow behaviour and fitted quite well with the Bingham plastic model in the studied range of concentrations. The specific multimodal iron ore samples with a broad size distribution indicated a substantial reduction in slurry viscosity, yield stress and improved solids loading compared to monomodal ones. The reduction in apparent viscosity at a given shear rate for the specific iron ore slurry sample was correlated to a distribution modulus ( $\psi$ ) derived from the Farris theory.

The application of small dosages (0.8-1\% $\mathrm{w} / \mathrm{w}$ of total solids) of two selective bio-additives extracted from Indian spinach (Basella alba) and Bellyache bush (Jatropha gossypifolia Linn) further reduced the Bingham viscosity and yield stress values through surface modification at higher solids loading. The increase in yield stress values with an increase in solids concentration was attributed to the formation of loosely packed flocs and immobilisation of water within them, thus increasing particle-particle and particle-fluid friction. The bio-additives may be able to improve the fluid mobility among the particles, thus reducing the internal friction constituting the flocs and facilitating the slurry flow with the application of a reasonably lesser shearing force. The study reveals that the blending of fines with coarse iron ore at a controlled particle size distribution supplemented by small dosages of low-cost additives may be employed for preparation and transportation of high-concentration iron ore slurry with improved pipe economics.
\end{abstract}

Keywords: iron ore, high concentration, rheology, bio-additives

\section{Introduction}

The Indian iron and steel industries have grown exponentially during the last two decades and it is expected that steel production will reach $180 \mathrm{M}$ t by 2019-2020. To meet such future demand, the run-of-mine (ROM) iron ore requirement would have to be over $500 \mathrm{M} \mathrm{t}$ per annum as against the present level of production of around $220 \mathrm{M} \mathrm{t}$ (Ministry of Steel 2017). Therefore, the long-term uninterrupted availability and supply of iron ore to processing sites at high rates is imperative for the growth of the Indian iron and steel industries, and the emphasis is now on adopting environmentally friendly transportation of ore through slurry pipelines in the coming years. The slurry pipelines will go a long way in reducing the problems of pollution and congested transportation networks in the mining areas. In order to reduce specific water consumption per ton of steel produced, the transportation of iron ore slurry at high solids concentration may be considered as an innovative solution. In such a scenario, the major Indian iron and steel players such as Steel Authority India Limited (SAIL), National Mineral Development Corporation (NMDC), TATA Steel, JSW Steel, Rashtriya 
Ispat Nigam Limited (RINL) and Jindal Steel \& Power Limited (JSPL) have ambitious future plans for installing and operating iron ore slurry pipelines to enhance steel production. Therefore, the preparation of a highly dispersed homogeneous suspension of iron ore particles in water as the carrier medium is essential prior to transporting the bulk slurry through pipelines. The rheological behaviour of the concentrated slurry requires careful investigation with respect to viscosity and other slurry flow parameters for negotiating the pumping power with minimum specific energy consumption (Govier \& Aziz 1972).

It is well known that the viscosity of a particulate suspension increases significantly with increases in solids loading and, by broadening the particle size distribution (PSD), the viscosity can be lowered substantially. Therefore, optimising PSD is an effective way to obtain high solids concentration with low viscosity (Barnes \& Hutton 1989; Zaman \& Moudgil 1998). Thus, polydispersity can give a lower viscosity at the same solids concentration, or allow a higher solids loading of particles at the equivalent viscosity (Farris 1968; Greenwood et al. 1998).

The relative viscosity of a bimodal and trimodal suspension can be described with the following equations:

$$
\begin{gathered}
\mu_{r}=h\left(\phi_{c}\right) h\left(\phi_{f}\right) \\
\mu_{r}=h\left(\phi_{c}\right) h\left(\phi_{m}\right) h\left(\phi_{f}\right)
\end{gathered}
$$

where:
$\mu_{r} \quad=$ relative viscosity.
$\emptyset_{c}=$ volume fraction of coarse-sized particles.
$\emptyset_{m}=$ volume fraction of medium-sized particles.
$\emptyset_{f} \quad=$ volume fraction of fine-sized particles.

Some researchers (Chang \& Powell 1994; Chong et al. 1971; Larson 1999; Maron \& Pierce 1956; Kitano et al. 1981; Krieger \& Dougherty 1959; Storms et al. 1990) have investigated the various important factors affecting the flow behaviour and predicting the viscosity of monomodal and polydispersed spherical particulate suspensions. For suspensions of polydispersed non-spherical particles, the influence of shape factor, particle density and surface roughness on maximum volume fraction and the interaction between the particles in static and dynamic flow situations have to be taken into account (Metzner 1985). Two major parameters, size ratio $(\lambda)$ and blend ratio $(\xi)$, may be employed to describe the polydispersive nature of suspension (Toivakka \& Eklund 1995). The size ratio $\lambda$ is defined as the ratio of the diameter of the large particles to that of the small particle diameter of the next particle class and is expressed as:

$$
\lambda=d_{l \arg e} / d_{\text {small }}
$$

In this case, the value of $d_{\text {large }}$ is taken as $d_{90}$, that is, $90 \%$ by volume of particles are smaller than this diameter and the value of $d_{\text {small }}$ is taken as $d_{10}$, that is, $10 \%$ by volume of particles are smaller than this diameter in a distribution. Similarly, the blend ratio $\xi$ is expressed as the fraction of a particle class in relation to the other classes (e.g. a system may have $10 \%$ coarse particles and $90 \%$ fine particles). These two parameters require manipulation to increase the maximum volume fraction in a suspension (Barnes 2000; Farris 1968; Hoffman 1992; Storms et al. 1990). In addition, to reduce slurry viscosity, the distribution modulus ( $\psi$ ) derived from Farris' work is strongly related to slurry concentration and the equation can be expressed as follows:

$$
\psi=\frac{\ln V_{l}}{\ln k}
$$

where:

$$
\begin{aligned}
& V_{l}=\text { volume fraction of liquid in the slurry. } \\
& k=d_{\text {small }} / d_{\text {large. }} .
\end{aligned}
$$


Further, it is conceived that the ability to achieve a high solids concentration can be determined by the ratio of the average separation $\beta$ of the large particles to the size of the fine material (Miller 1993). In the present investigation, this parameter for the iron ore sample was evaluated by using the following equation:

$$
\beta=\left[\left(K_{V} / \phi_{b}\right)^{\frac{1}{3}}-1\right] d_{b}
$$

where:

$K_{V}=$ column shape factor (i.e. $\pi / 6$ ).

$\phi_{b}=$ volume fraction of coarse iron ore particles.

$d_{b}=$ median diameter of coarse iron ore particles.

A limited study on the rheological behaviour of a concentrated iron ore slurry has been cited in the literature. Jennings (1969) investigated the flow behaviour of aqueous suspension of hematite slurry samples containing finely divided hematite particles $(<45 \mu \mathrm{m})$. The slurry flow behaviour was markedly improved in the presence of lignosulfonate dispersant with the volume concentration up to $40 \%$. Studies carried out by Vieira and Peres (2012) indicated that the dosage of chemical reagents such as polyacrylic acids, silicone acrylate copolymer, citric acid and sodium hexametaphosphate reduced the apparent viscosity and fluid consistency index of iron ore concentrate samples at SAMARCO's concentrator. The flow behaviour of the slurry samples were fitted to Bingham and Herschel-Bulkley models.

Moraes et al. (2013) in their review article have categorically mentioned the use of sodium humate, polyacrylic acid, polymethacrylic acid, sodium hydroxide and nitric acid to improve the dispersion stability of iron ore fines/hematite slurry. The stability of ultrafine iron ore slurry at low solids concentration (7.5\%) was studied by Abro et al. (2010) and the optimal dosage of chemical dispersants with respect to slurry $\mathrm{pH}$, stirring speed and time were evaluated. Melorie and Kaushal (2017) indicated that the dosages of sodium hexametaphosphate reduced the shear stress and viscosity values of iron ore fines slurry in slurry concentration range of $18.8-25.8 \%$ by volume. Sahoo et al. (2017) investigated the influence of microwave energy on apparent viscosity of Indian iron ore slurry and observed that the tested samples were very much affected by particle size, solids concentration and microwave exposure time. Some studies on improving the energy efficiency on slurry transport depicting the effect of pipe diameter, solids concentration and particle size has been investigated (Aziz \& Mohamad 2013; Hashemi \& Sanders 2014; Ihle et al. 2014; Parida et al. 2000; Yildiz et al. 2014; Wu et al. 2010).

Considering the limited literature on preparation and rheological characterisation of highly loaded iron ore fines/concentrate slurry, this paper investigates the effect of manipulating the particle size distribution and dosage of selective bio-additives on preparation and then evaluating the flow behaviour of iron ore slurry in a solids concentration range of $60-75 \%$ by mass.

\section{Experimental}

\subsection{Characterisation studies}

The iron ore samples used for the study were procured from Essar Steel captive mines in the Barbil area, Keonjhar district of Odisha (situated in the eastern part of India). Initially, the lumpy ores with 8-10 mm sizes were ground in a ball mill with different grinding times and three distinct fine and coarse powder particle size ranges, i.e. $<45 \mu \mathrm{m}, 45-150 \mu \mathrm{m}$ and $45-300 \mu \mathrm{m}$, were obtained by screening using standard sieves. The average true density of the iron ore sample determined by laboratory tests was found to be $4,508 \mathrm{~kg} / \mathrm{m}^{3}$. Three representative iron ore samples with monomodal, bimodal and multimodal characteristics were prepared from the aforementioned three distinct particle size ranges and labelled as S-1, S-2 and S-3. Sample S-1 contains only finer fraction $(<45 \mu \mathrm{m})$ particles. Samples S-2 and S-3 were prepared by blending the finer fraction $(<45 \mu \mathrm{m})$ with the other two coarse fractions $(45-150 \mu \mathrm{m}$ and $45-300 \mu \mathrm{m}$ size particles) 
separately in a fixed blend ratio (mass ratio) of 4:1. The PSD of these three representative samples was determined by using a HORIBA LA-960 laser scattering particle size distribution analyser. The samples were first dispersed in distilled water and tetrasodium pyrophosphate was used to keep the solution afloat. An internal ultrasound probe urges sample dispersion and the ultrasound wave exposure time was set for about three minutes to avoid formation of any agglomerates of limestone particles. The PSD of the three iron ore samples are given in Table 1 and the chemical composition of the sample is given in Table 2 . The instantaneous $\mathrm{pH}$ of iron ore slurry was found to be 7.33 at a slurry concentration of $70 \%$ by mass and $\mathrm{pH}$ stabilised after five hours of mixing and remained alkaline with a $\mathrm{pH}$ value of 7.44.

Table 1 Particle size distribution of the three representative iron ore samples used for the study

\begin{tabular}{llll}
\hline Diameter on cumulative \% & S-1 $(\mu \mathrm{m})$ & S-2 $(\mu \mathrm{m})$ & S-3 $(\mu \mathrm{m})$ \\
\hline$d_{10}$ & 3.52 & 4.73 & 3.26 \\
$d_{50}$ & 9.11 & 12.84 & 10.62 \\
$d_{90}$ & 25.06 & 55.2 & 77.23 \\
\hline
\end{tabular}

Table 2 Chemical analysis of mine sample

\begin{tabular}{lllll}
\hline Mine name & $\mathrm{Fe}(\%)$ & $\mathrm{SiO}_{2}(\%)$ & $\mathrm{Al}_{2} \mathrm{O}_{3}(\%)$ & LOI (\%) \\
\hline $\begin{array}{l}\text { Kamaljeet Singh Ahluwalia (KJSA) } \\
\text { Barbil, Keonjhar and Odisha }\end{array}$ & 64.08 & 2.54 & 1.14 & 2.3 \\
\hline
\end{tabular}

\subsection{Maximum static settled concentration (sedimentation) test}

In order to ascertain the limiting concentration of the iron ore slurry samples, static settling tests were carried out in the laboratory for the three representative samples following a standard procedure. Initially, slurries were prepared in a mass concentration range of $55-75 \%$ and poured into standard graduated glass cylinders with a $250 \mathrm{ml}$ capacity. Then the slurries were allowed to settle in the cylinders for about 72 hours, after which no further settling occurs. By noting down the interface readings between settled slurry and free water at the top, the maximum settled concentration was computed using the following relationship:

$$
C_{W \max }=\frac{M_{S}}{M_{S}+M_{W}}
$$

where:

$$
\begin{aligned}
& M_{S}=\text { mass of solids in the settled mass. } \\
& M_{W}=\text { mass of water present in the settled mass. }
\end{aligned}
$$

\subsection{Rheological measurements}

The rheological experiments for the three iron ore samples in the slurry concentration range of $60-75 \%$ by mass were conducted using a ThermoFisher Scientific HAAKE ${ }^{\mathrm{TM}}$ RheoStress ${ }^{\mathrm{TM}} 1$ Rheometer. The cup and bob sensor system Z comprising of a collapsible beaker Z43 and rotor Z41 (radius: $20.71 \mathrm{~mm}$ ) was used for rheological measurements of the three samples S-1, S-2 and S-3. Slurries prepared at the desired concentrations were subjected to rheological measurements under controlled shear rates $\left(0\right.$ to $\left.300 \mathrm{~s}^{-1}\right)$ and at a room temperature of $30^{\circ} \mathrm{C}$. By following the same procedure, rheological measurements of iron ore samples were carried out with the application of small dosages $(0.6-1 \% \mathrm{w} / \mathrm{w}$ of total solids) of two selective bio-additives extracted from Indian spinach (Basella alba) and Bellyache bush (Jatropha gossypifolia Linn). 


\section{$3 \quad$ Results and discussion}

\subsection{Effects of blending coarse fractions with finer fractions on maximum static settled concentration ( $C_{W \text {-max }}$ ) of iron ore slurry}

The maximum settled concentration test data for the three samples at different mass concentrations are presented in Table 3. From the test data as indicated in Table 3, the $C_{W \text {-max }}$ values exhibit an increasing trend with the increase in slurry concentration from 55 to $75 \%$ by mass for the three slurry samples. This may be due to the gradual decrease of settling rates and minimisation of voids with the increase in initial slurry concentration from 55 to $75 \%$ by mass. However, the blended samples S-2 and S-3 with bimodal and multimodal particle size distributions influenced the maximum static settled concentration data very much, as observed in Table 2 . The sample $S-2$ indicated a maximum value of $C_{W \text {-max }}$ when compared to the other two samples S- 1 and S-3 in the studied range of concentrations. The plot of $C_{W \text {-max }}$ versus $\beta / d_{50 \text {-fine }}$ at solids mass concentrations of 55, 60, 65, 70 and $75 \%$ for the three samples (S-1 to S-3) is indicated in Figure 1.

Table 3 Maximum static settled concentration (sedimentation) test data for iron ore samples (S-1 to $\mathrm{S}-3)$

\begin{tabular}{llllll}
\hline Iron ore samples & $C_{w}=55 \%$ & $C_{w}=60 \%$ & $C_{w}=65 \%$ & $C_{w}=70 \%$ & $C_{w}=75 \%$ \\
\hline S-1 (monomodal) & 72.37 & 73.62 & 74.71 & 75.68 & 76.53 \\
S-2 (bimodal) & 73.34 & 74.53 & 75.58 & 76.46 & 77.22 \\
S-3 (multimodal) & 72.85 & 74.12 & 75.14 & 76.1 & 76.94 \\
\hline
\end{tabular}

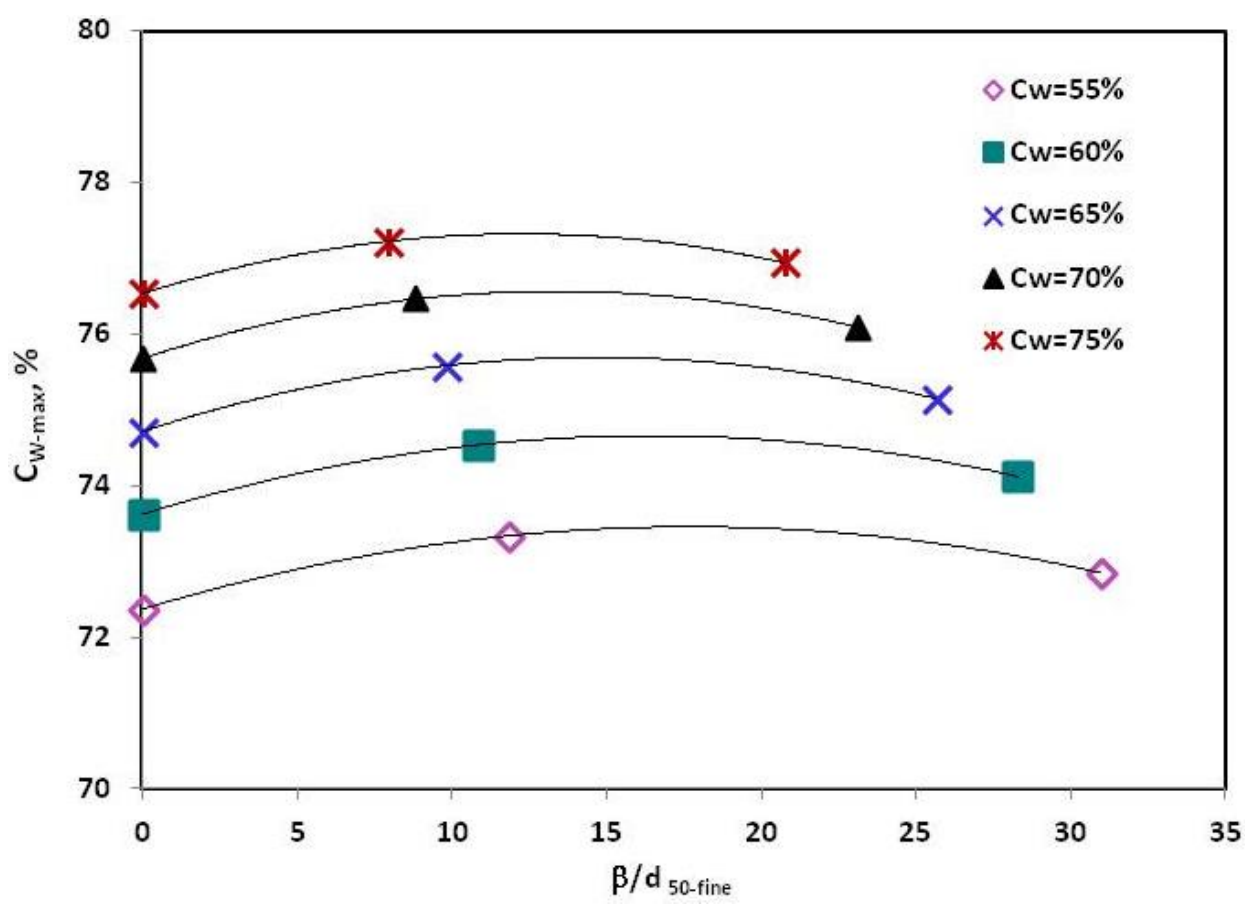

Figure $1 C_{W-\max }$ versus $\beta / d_{50-f}$ for the iron ore slurry samples (S-1 to $\mathrm{S}-3$ )

It is observed from Figure 1 that the parameter $\beta / d_{50 \text {-fine }}$ strongly influences the $C_{W \text {-max }}$ values with bimodal and multimodal distributions of particle sizes at a given solids concentration of iron ore slurry. This may be because the selected fine iron ore particles can readily fill the void spaces between the selected coarse particles and, therefore, higher iron ore loading can be obtained. Thus, as the size of the iron ore is reduced, the excluded volume effect becomes increasingly important and enhances the $C_{W \text {-max }}$ values at these concentrations for samples S-2 and S-3. With the increase in the solids concentration of the slurry mixture 
from 55 to $75 \%$ by mass, the solid volume of fine and coarse particles increases and, consequently, the available void volume increases. Hence, the increase in $C_{W \text {-max }}$ values observed for the two blended iron ore samples may be attributed to the enhancement of packing possibilities as more and finer particles tend to fill the voids as indicated in Table 3. Sample S-3, with multimodal characteristics, did not provide the highest maximum iron ore loading as expected. This may be due to the fact that the ultrafine and fine particles may form agglomerates or compact structures within the voids between the relatively coarse particles and reduce the free space available for packing.

\subsection{Rheological behaviour of iron ore slurry}

The rheological data in terms of shear stress $(\tau)$ and shear rate $(\dot{\gamma})$, in the slurry concentration range of $60-75 \%$ by mass for the iron ore sample S-1 is plotted in Figure 2. The flow characteristics of the slurry indicated Bingham plastic flow behaviour which is observed from the shape of the rheogram $\tau-\dot{\gamma}$ and the flow pattern is non-Newtonian at these solids concentrations. It is further observed that the shear stress values increased with the increase in mass concentration and, hence, the viscosity of the slurry increased with the increase in solids loading. The $R^{2}$ coefficients for the fit of different curves were found to be in the range of 0.9848 to 0.9983 . Thus, the Bingham plastic model fitted very well to the experimental data at these concentrations, which may be written as:

$$
\tau=\tau_{O}+\eta_{p} \dot{\gamma}
$$

where:

$$
\begin{aligned}
\tau & =\text { shear stress }(\mathrm{Pa}) . \\
\dot{\gamma} & =\text { shear rate }\left(\mathrm{s}^{-1}\right) . \\
\tau_{0} & =\text { yield stress }(\mathrm{Pa}) . \\
\eta_{p} & =\text { plastic viscosity (Pas). }
\end{aligned}
$$

A similar type of flow behaviour was observed for the other two iron ore samples (S-2 and S-3). However, these are not given here due to paucity of space. The rheological parameters $\tau_{0}(\mathrm{~Pa})$ and $\eta_{p}$ (Pas) values obtained for the three samples in the studied range of concentrations are presented in Table 4.

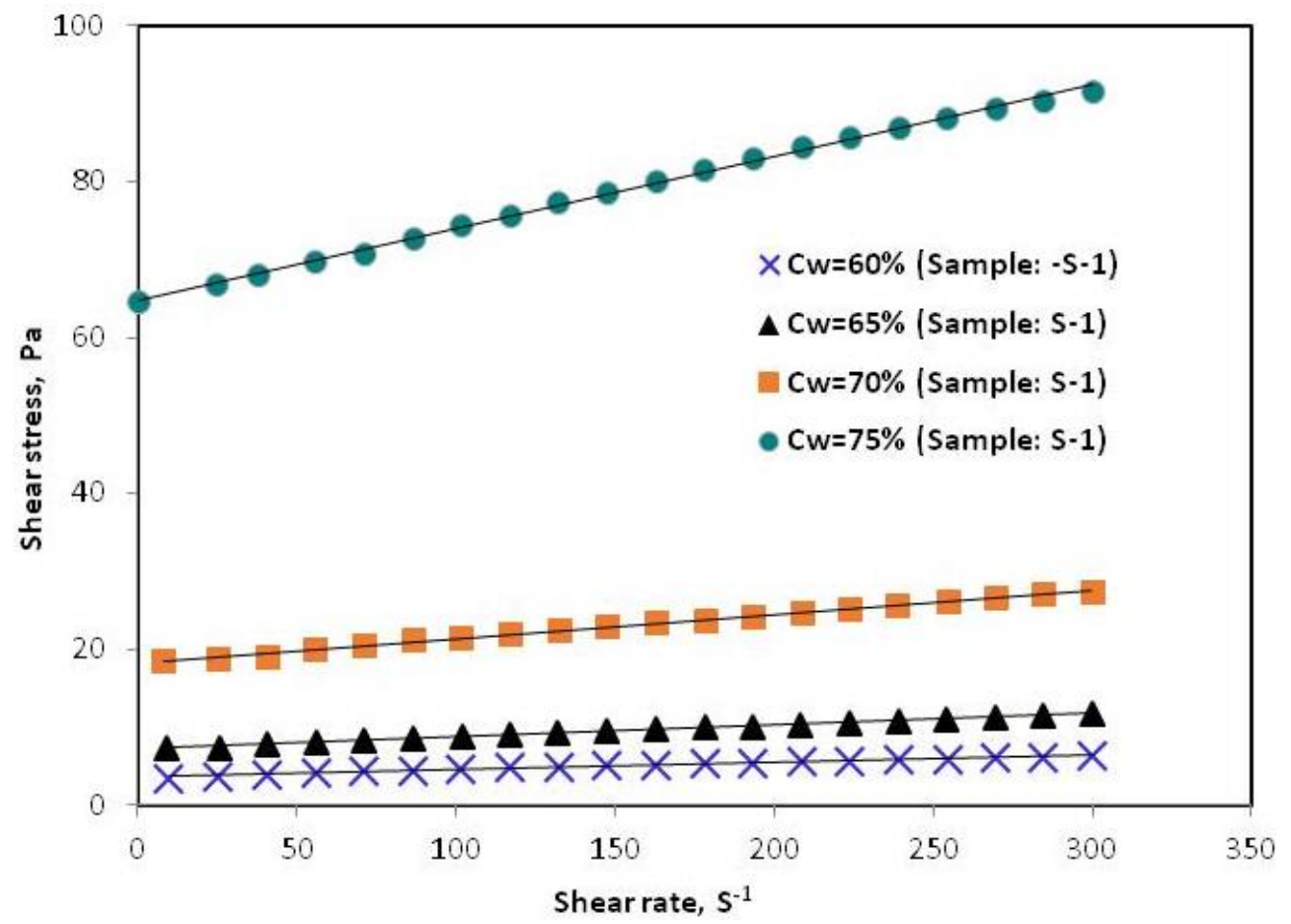

Figure 2 Rheogram of iron ore slurry at different concentrations by mass (sample: S-1) 
Table 4 Rheological parameters for the iron ore slurry samples at different $C_{w} \%$

\begin{tabular}{llllll}
\hline $\begin{array}{l}\text { Iron ore } \\
\text { samples }\end{array}$ & $\begin{array}{l}\text { Rheological } \\
\text { parameters }\end{array}$ & $C_{w}=60 \%$ & $C_{w}=65 \%$ & $C_{w}=70 \%$ & $C_{w}=75 \%$ \\
\hline \multirow{3}{*}{ S-1 } & $\tau_{0}, \mathrm{~Pa}$ & 3.55 & 7.2 & 18.3 & 64.85 \\
& $\eta_{p}, \mathrm{Pas}$ & 0.0093 & 0.015 & 0.031 & 0.092 \\
\multirow{2}{*}{ S-2 } & $\tau_{0}, \mathrm{~Pa}$ & 2.76 & 4.37 & 10.8 & 26.96 \\
& $\eta_{p}, \mathrm{Pas}$ & 0.009 & 0.014 & 0.025 & 0.057 \\
& $\tau_{0}, \mathrm{~Pa}$ & 4.06 & 5.82 & 12.16 & 28.92 \\
S-3 & $\eta_{p}, \mathrm{Pas}$ & 0.0091 & 0.016 & 0.032 & 0.066 \\
\hline
\end{tabular}

\subsubsection{Effect of fine-coarse blending on yield stress of iron ore slurry}

It is observed from Table 4 that the rheological parameters were very much influenced by blending the finer fractions with coarse fractions at a fixed blend ratio of 4:1. The Bingham yield stress increased sharply beyond a slurry concentration of $70 \%$ by mass for sample S-1. The other two samples, with bimodal and multimodal particle size distribution, indicated lesser yield stress as compared to sample S-1 in the studied range of slurry concentrations $\left(C_{W}=60-75 \%\right)$. This is because at a higher solid concentration with smaller inter-particle distance, sample S-1 produces an increased attractive force and certain limiting shearing force is required to break down the loosely packed flocs and overcome the internal friction among the fine particles to initiate the flow. By blending the fine particles with fractions of the coarse particles, samples S-2 and S-3 incurred lower yield stress due to a reduction in the number of flocculated structures. Bentz et al. (2012) and Shivaram et al. (2013) made similar observations while characterising the flow behaviour of fine cement with coarse fly ash slurry and ultrafine mallee bio-char slurry respectively.

\subsubsection{Effect of particle size distribution on apparent viscosity of iron ore slurry}

The plot of apparent viscosity versus shear rate at slurry mass concentrations of $75 \%$ for the three iron ore samples S-1, S-2 and S-3 is presented in Figure 3.

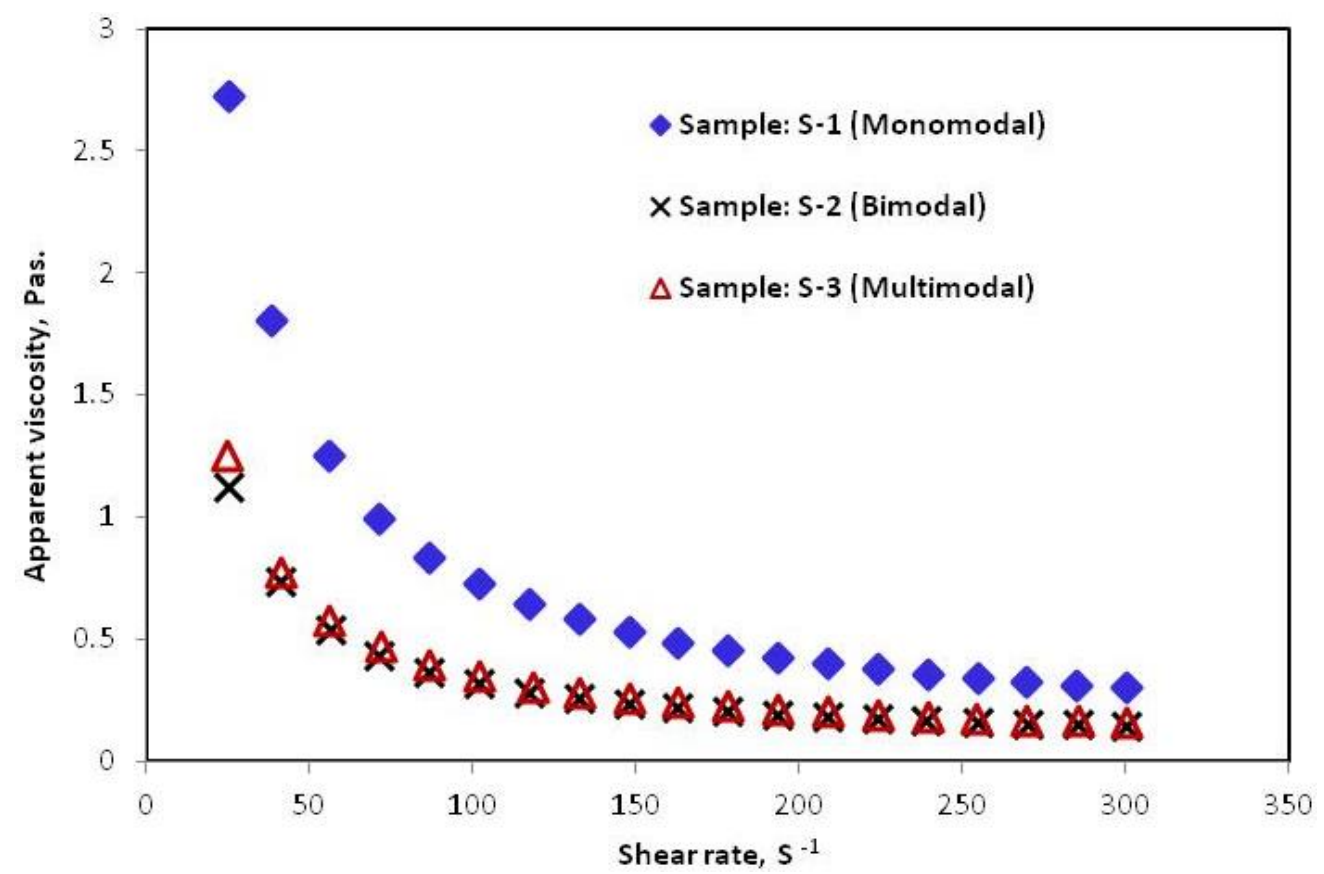

Figure 3 Effect of particle size distribution on apparent viscosity of iron ore slurry at high solids concentration $\left(C_{W}=75 \%\right)$ 
Figure 3 shows that samples S- 2 and S-3 with bimodal and multimodal packing characteristics influenced the apparent viscosity as compared to monomodal sample S-1 in the studied range of shear rates. An appreciable amount of reduction in apparent viscosity was observed for S-2 and S-3 in comparison to S-1 at a slurry concentration of $75 \%$ by mass. The reduction in viscosity for S-2 and S-3 may be due to the fact that at a given solids volume fraction, the blending of fines with coarse particles in a fixed blend ratio attains a bidisperse suspension. The viscosity is greatly influenced by size ratio $(\lambda)$ which is defined as the diameter ratio of large to small particles and is given by $\lambda=d_{\text {large }} / d_{\text {small. }}$. The plot of size ratio versus apparent viscosity for the three iron ore samples in the slurry concentration range of $70-75 \%$ by mass at shear rates of $300 \mathrm{~s}^{-1}$ is shown in Figure 4 .

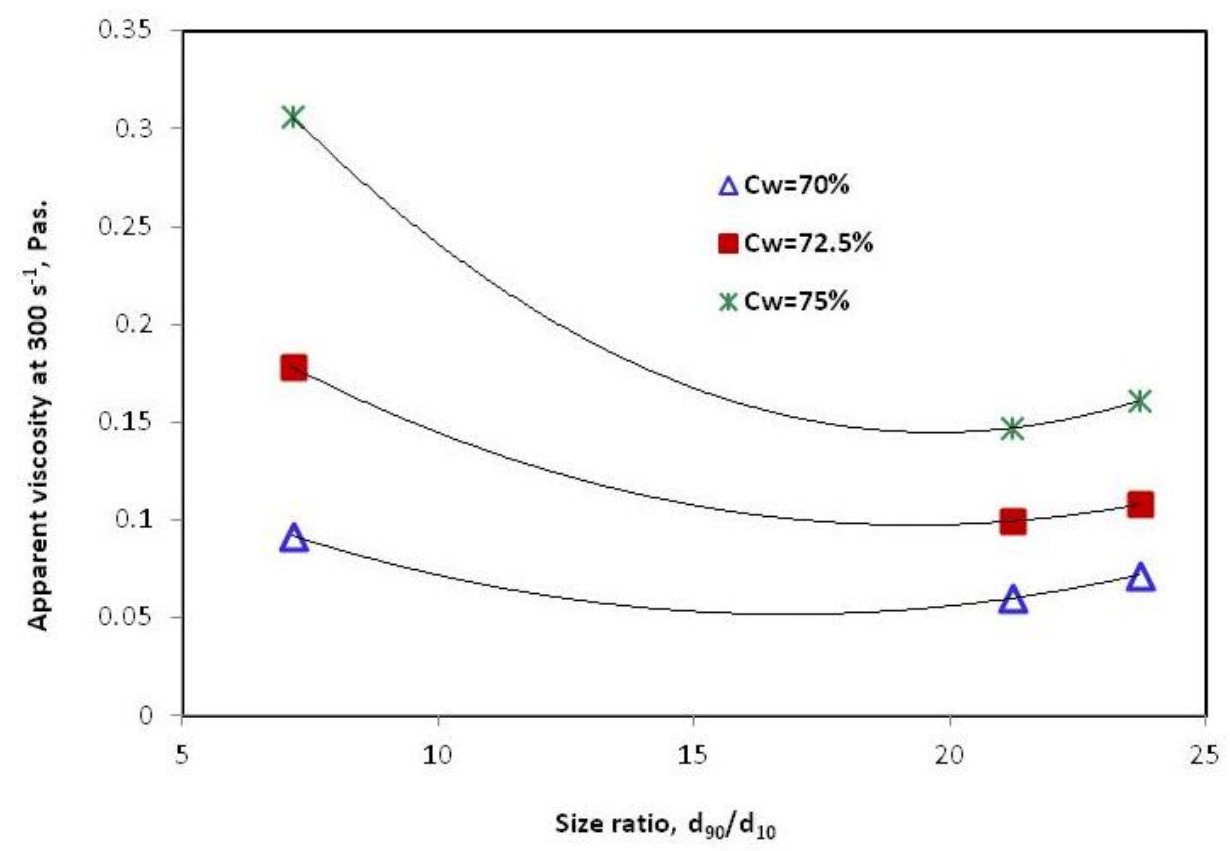

Figure 4 Effect of size ratio $(\lambda)$ on apparent viscosity for the three iron ore samples at shear rate of $300 \mathrm{~s}^{-1}$

Figure 4 indicates that the apparent viscosity increased with a decrease in size ratio $(\lambda)$ for the three iron ore samples in a slurry concentration range of $70-75 \%$ by mass. The increase in apparent viscosity at a given shear rate may be due to stronger interparticle attractions in a slurry sample with finer particles at a specified concentration. As revealed in the literature, minimum slurry viscosity can be achieved with size ratio of greater than 7 (Metzner 1985) and is valid for bi, tri and tetramodal PSD. Thus, the favourable diameter ratios of samples S-2 and S-3 with bimodal and multimodal characteristics in our study led to lowering the viscosities. The reduction in Bingham plastic viscosity $\left(\eta_{p}\right)$, at high solids concentration observed for the iron ore samples was further substantiated by correlating the distribution modulus $(\psi)$ derived from the Farris theory. The distribution modulus $(\psi)$ for the samples S-1, S-2 and S-3 at high solids concentrations were computed to optimise the slurry viscosity with respect to PSD of a given iron ore sample. The Bingham viscosity values for the samples obtained experimentally were plotted against the computed values of distribution modulus $(\psi)$ in a slurry concentration range of $(70-75 \%)$ and are presented in Figure 5.

The data points at each concentration were fitted to a polynomial curve and the resulting equation corresponding to these concentrations can be expressed as:

$$
\eta_{p_{i}}=a \psi_{i}^{2}-b \psi_{i}+c
$$

where:

$$
\begin{array}{ll}
\eta_{p i} & =\text { Bingham viscosity (Pas). } \\
\psi_{l} & =\text { distribution modulus. } \\
a, b, c & =\text { curve fitting parameters. }
\end{array}
$$




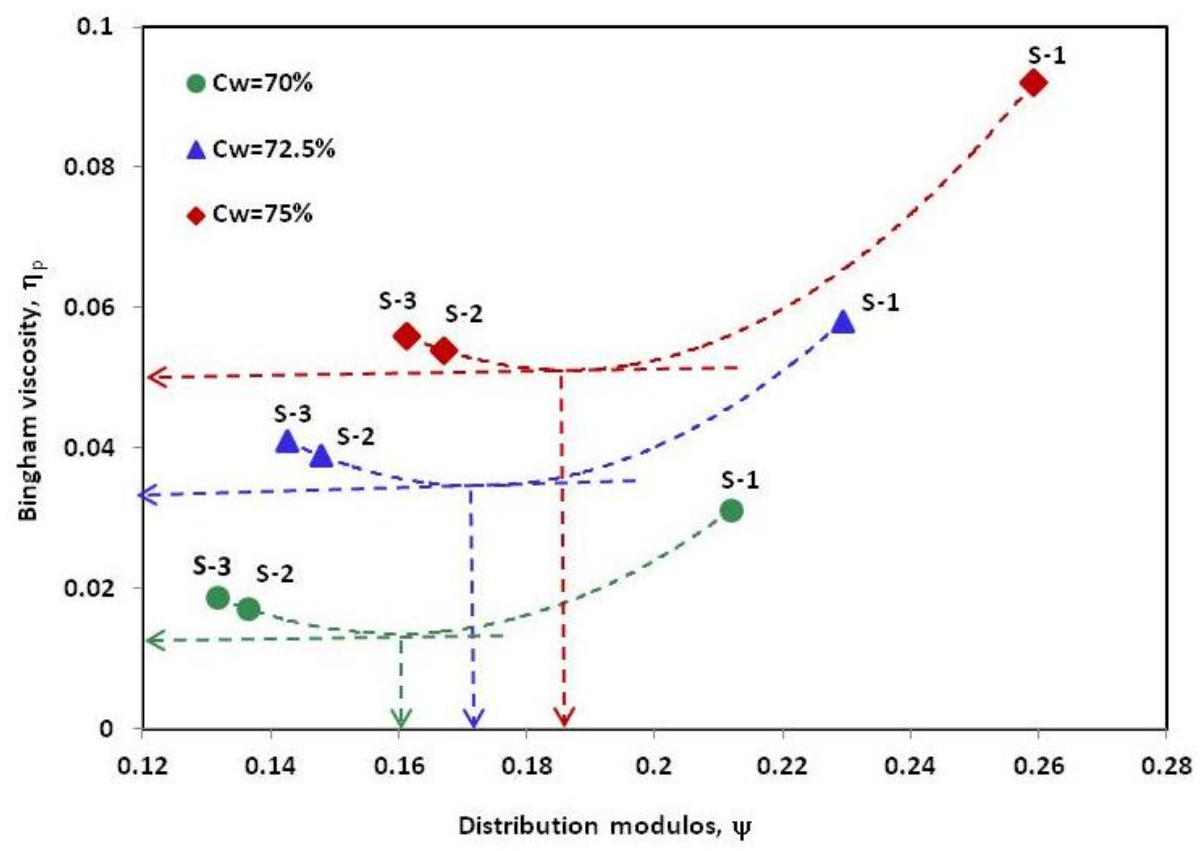

Figure 5 Experimental values of Bingham viscosity versus distribution modulus $(\psi)$ for the three iron ore samples $\left(C_{w}=70,72.5\right.$ and $\left.75 \%\right)$

Using Equation 8, the minimum or best value of the $\psi$ was evaluated to be in the range of 0.161 to 0.186 for the iron ore samples. The sample S- 2 with a $\psi$ value of 0.167 indicated near-minimum viscosity at the highest solids concentration of $75 \%$ and achieved maximum packing density as discussed earlier. Therefore, the distribution modulus value is in remarkable agreement with the theoretical prediction of Farris where the optimum $\psi$ values were in the range of 0.174 to 0.221 for the set of coal water slurries having a ratio of a largest particle diameter $\left(d_{\text {large }}\right)$ in the distribution to the smallest particle diameter $\left(d_{\text {small }}\right)$ in a broad distribution of coal particles (Farris 1968).

\subsubsection{Effect of bio-additives dosages on apparent viscosity of iron ore slurry}

The plot of apparent viscosity versus shear rate data obtained for samples S-1, S-2 and S-2 with small dosage ( $0.8 \% \mathrm{w} / \mathrm{w}$ of total solids) of two selective bio-additives extracted from Indian spinach and Bellyache bush at a solids concentration of $75 \%$ by mass is presented in Figure 6 . It was observed from the plot that the selective additives influenced the flow behaviour of the iron ore slurry and could able to reduce the apparent viscosity in the studied range of shear rate. The lignans, saponins and tannins content in these two bio-additives probably helps in surface modification through their lubricating effects, and act as stabilising agents for iron ore slurry at higher solids loading. Bellyache bush extract was found to be more effective than Indian spinach in reducing the yield stress and viscosity of iron ore slurry. The percentage reduction in yield stress and Bingham viscosity values for $\mathrm{S}-2$ was found to be in the range of $8.4-14.7 \%$ and $12.3-15.4 \%$ respectively with the two bio-additive dosages $(0.8 \% \mathrm{w} / \mathrm{w}$ of total solids) at a slurry concentration of $75 \%$ by mass. 


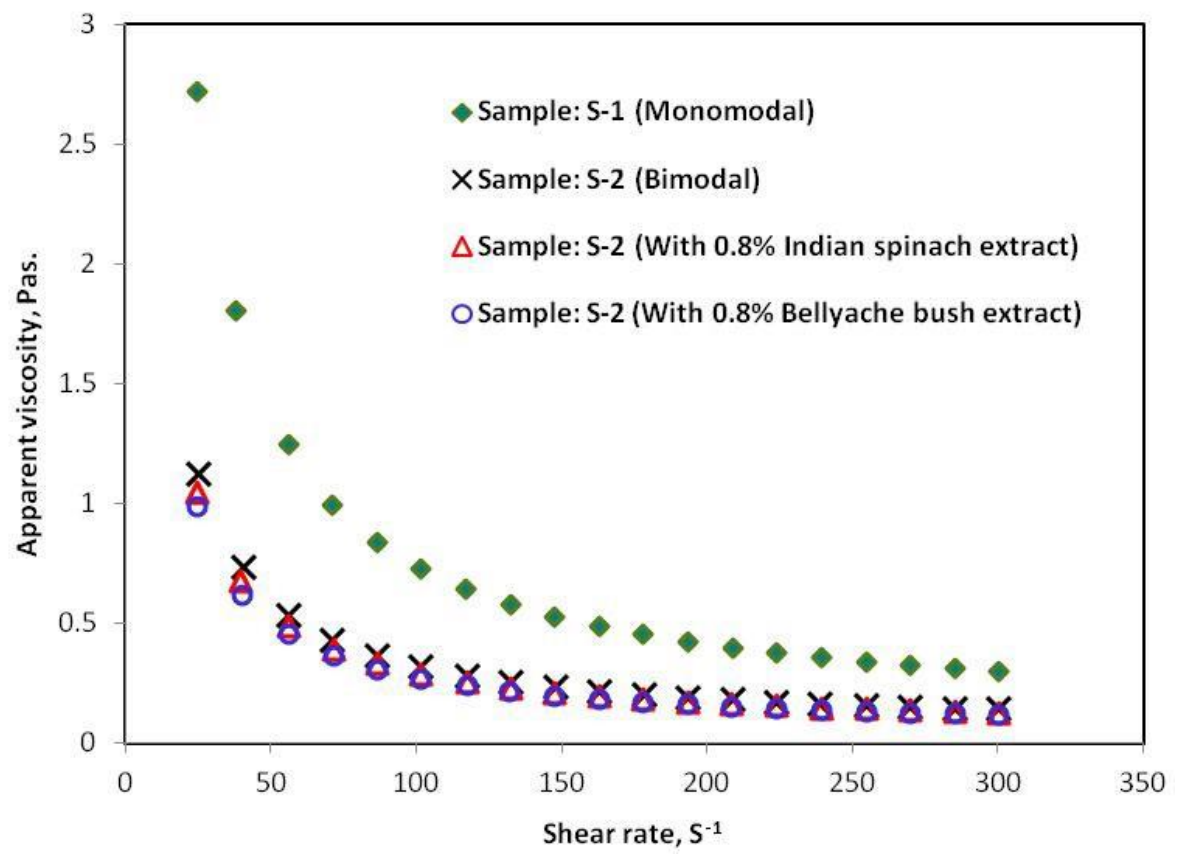

Figure 6 Effect of selective bio-additives on apparent viscosity of iron ore slurry

\section{$4 \quad$ Conclusions}

The iron ore slurry samples with and without the addition of bio-additives exhibited viscoplastic properties and the flow behaviour could be characterised by the Bingham plastic model in the studied range of slurry concentrations ( $C_{W}=60-75 \%$ by mass). The maximum amount of reduction in viscosity and yield stress values obtained in respect of sample S-2 could be explained by correlating the viscosity to particle size ratio $\left(\lambda=d_{\text {large }} / d_{\text {small }}\right)$ and volume fraction of solids. The sample S- 2 with a distribution modulus $(\psi)$ value of $\sim 0.17$ indicated near-minimum viscosity and maximum packing density, which was in reasonable agreement with the Farris (1968) theoretical prediction. The results thus illustrated that the magnitude of viscosity reduction and maximum solids concentration could be achieved by controlling the diameter ratio and composition of fine to coarse iron ore particles. The dosing of selective bio-additives extracted from Indian spinach and Bellyache bush in relatively small concentrations ( $0.8-1 \%$ of total solids) with iron ore slurry may be considered a viable option for efficient and economic pipeline transport by lowering pumping costs and energy.

\section{Acknowledgement}

The authors are gratefully indebted to Mr SK Mishra, Acting Director, CSIR-Institute of Minerals and Materials Technology, Bhubaneswar for giving permission to publish this paper and thank the Ministry of Steel, New Delhi and the National Mineral Development Corporation, Hyderabad, India for their financial support of the investigation.

\section{References}

Abro, MI, Pathan, AG, Andreas, B \& Mallah, AH 2010, 'Effect of various parameters on the dispersion of ultra fine iron ore slurry. Part-2', Pakistan Journal of Analytical \& Environmental Chemistry, vol. 10, no. 2, pp. 18-22.

Aziz, AIAA \& Mohamad, HI 2013, 'A study of the factors affecting transporting solid-liquid suspension through pipelines', Open Journal of Fluid Mechanics, vol. 3, pp. 152-162.

Barnes, HA, Hutton, JF \& Walters K 1989, An Introduction to Rheology, Elsevier, Amsterdam, p. 122.

Barnes, HA 2000, A Handbook of Elementary Rheology, University of Wales Institute of Non-Newtonian Fluid Mechanics, Aberystwyth, p. 123.

Bentz, DP, Ferraris, CF \& Galle, MA 2012, 'Influence of particle size distribution on yield stress and viscosity of cement-fly ash pastes', Cement and Concrete Research, vol. 42, no. 2, pp. 404-409.

Chang, C \& Powell, RL 1994, 'Effect of particle size distributions on the rheology of concentrated bimodal suspensions', Journal of Rheology, vol. 38, no. 1, pp. 85-98. 
Chong, JS, Christiansen, EB \& Baer, AD 1971, 'Rheology of concentrated suspensions', Journal of Applied Polymer Science, vol. 15, pp. 2007-2021.

Farris, JR 1968, 'Prediction of the viscosity of multimodal suspensions from unimodal viscosity data', Transaction of the Society of Rheology, vol. 12, no. 2, pp. 281-301.

Govier, GW \& Aziz, K 1972, The Flow of Complex Mixtures in Pipes, Van Nostrand Reinhold Company Publishing, New York.

Greenwood, R, Luckham PF \& Gregory, T 1998, 'Minimizing the viscosity of concentrated dispersions by using bimodal particle size distributions', Colloids \& Surface A: Physicochemical \& Engineering Aspects, vol. 144, no. 1-3, pp. 139-147.

Hoffman, RL 1992, 'Factors affecting the viscosity of unimodal and multimodal colloidal dispersions', Journal of Rheology, vol. 36, no. 5, http://sor.scitation.org/doi/abs/10.1122/1.550324

Jennings, HYJ 1969, 'Effect of surfactants on the rheology of hematite slurries', Journal of the American Oil Chemists' Society, vol. 46, no. 12 , pp. 642-644.

Hashemi, SA \& Sanders RS 2014, 'Specific energy consumption and optimum operating condition for coarse-particle slurries', Power Technology, vol. 262, pp. 183-187.

Ihle, C, Tamburrino, A \& Montserrat, S 2014, 'Computational modeling for efficient long distance ore transport using pipelines', Minerals Engineering, vol. 63, pp. 73-80.

Kitano, T, Kataoka, T \& Shirota, T 1981, 'An empirical equation of the relative viscosity of polymer melts filled with various inorganic fillers', Rheologica Acta, vol. 20, pp. 207-209.

Krieger, IM \& Dougherty, TJ 1959, 'A mechanism for non-Newtonian flow in suspensions of rigid spheres', Transaction of the Society of Rheology, vol. 3, pp. 137-152.

Larson, RG 1999, The Structure and Rheology of Complex Fluids, Oxford University Press, Oxford.

Maron, SH \& Pierce, PE 1956, 'Application of Ree-Eyring generalized flow theory to suspensions of spherical particles', Journal of Colloid Science, vol. 11, p. 80.

Melorie, AK \& Kaushal, DR 2017, 'Experimental investigation of the effect of chemical additives on the rheological properties of highly concentrated iron ore slurries', KONA Powder and Particle Journal, vol. 35, pp. 1-14.

Metzner, AB 1985, 'Rheology of suspensions in polymeric liquids', Journal of Rheology, vol. 29, no. 6, pp. 739-775.

Miller, BG 1993, The Development of Coal-based Technologies for Department of Defense Facilities, Semi-annual technical progress report for the period 3/28/1993 to 9/27/1993, Consortium for Coal-Water Slurry Fuel Technology, Pennsylvania State University, University Park, Pennsylvania.

Moraes, SL, Lima, JRB \& Neto, JBF 2013, 'Influence of dispersants on the rheological and colloidal properties of iron ore ultrafine particles and their effect on the pelletizing process-a review', Journal of Materials Research and Technology, vol. 2, no. 4, pp. 386-391.

Ministry of Steel 2017, National Steel Policy 2017, Ministry of Steel, Government of India, New Delhi, http://steel.gov.in/sites /default/files/draft-national-steel-policy-2017.pdf

Parida, A, Mishra, RN, Senapati, PK, Rohella, RS \& Murty, JS 2000, 'Transportation studies and pipeline design for disposal of iron ore slime', in GV Rao, B Das, SRS Sastri \& HS Ray (eds), Proceedings of the National Seminar on Recent Techniques in Mineral Processing Wastes Environment Management, Allied Publishers, New Delhi, pp. 280-285.

Sahoo, BK, Das, TK, Gupta, A, De, S, Carsky, M \& Meikap, BC 2017, 'Application of response surface analysis to iron ore slurry rheology using microwave pre-treatment', South African Journal of Chemical Engineering, vol. 23, pp. 81-90.

Shivaram, P, Leong, YK, Yang, H \& Zhang, DK 2013, 'Flow and yield stress behaviour of ultrafine Mallee biochar slurry fuels: the effect of particle size distribution \& additives' Fuel, vol. 104, pp. 326-332.

Storms, RF, Ramarao, BV \& Weiland, RH 1990, 'Low shear rate viscosity of bimodal dispersed suspensions', Powder Technology, vol. 63, pp. 247-259.

Toivakka, M \& Eklund, D 1995, 'Prediction of suspension rheology through particle motion simulation', Proceedings of the Advanced Coating Fundamental Symposium, Atlanta, pp. 161-177.

Vieira, MG \& Peres, AEC 2012, 'Effect of reagents on the rheological behaviour of an iron ore concentrate slurry', International Journal of Mining Engineering and Mineral Processing, vol. 1, no. 2, pp. 38-42.

Wu, J, Graham, L, Wang, S \& Parthasarathy, R 2010, 'Energy efficiency slurry holding and transport', Minerals Engineering, vol. 23, no. 9, pp. 705-712.

Yildiz, B, Sakarya, BA \& Ger, MA 2014, 'Optimum design of slurry pipelines by genetic algorithm', Civil Engineering and Environmental Systems, vol. 31, no. 4, pp. 311-330.

Zaman, AA \& Moudgil, BM 1998, 'Rheology of bidisperse aqueous silica suspensions: a new scaling method for the bidisperse viscosity', Journal of Rheology, vol. 42, pp. 21-39. 
